\title{
Lifetime of high-order thickness resonances of thin silicon membranes
}

A. A. Maznev ${ }^{\text {a }}$, F. Hofmann ${ }^{\text {b }}$, J. Cuffe $^{\text {c }}$, J. K. Eliason ${ }^{\text {a }}$, K. A. Nelson ${ }^{\text {a }}$

a Department of Chemistry, Massachusetts Institute of Technology, Cambridge, Massachusetts 02139, USA

${ }^{\mathrm{b}}$ Department of Engineering Science, University of Oxford, Parks Road, Oxford, OX1 3PJ, United Kingdom

${ }^{c}$ Department of Mechanical Engineering, Massachusetts Institute of Technology, Cambridge, Massachusetts 02139, USA

\begin{abstract}
Femtosecond laser pulses are used to excite and probe high-order longitudinal thickness resonances at a frequency of $\sim 270 \mathrm{GHz}$ in suspended Si membranes with thickness ranging from 0.4 to 15 micrometers. The measured acoustic lifetime scales linearly with the membrane thickness and is shown to be controlled by the surface specularity which correlates with roughness characterized by atomic force microscopy. Observed $Q$-factor values up to 2400 at room temperature result from the existence of a local maximum of the material $Q$ in the sub-THz range. However, surface specularity would need to be improved over measured values of $\sim 0.5$ in order to achieve high $Q$ values in nanoscale devices. The results support the validity of the diffuse boundary scattering model in analyzing thermal transport in thin Si membranes.
\end{abstract}




\section{Introduction}

A thickness resonance of a suspended plate is perhaps the simplest kind of solid state acoustic oscillator. Thickness resonances have long been used in quartz oscillators, and state-of-the art thin film resonators using thickness resonances of suspended thin films and operating at frequencies up to a few $\mathrm{GHz}$ are widely used in telecommunications electronics [1]. In recent years, ultra-short laser pulses have been used to excite thickness resonances of ultra-thin membranes in the sub-THz frequency range [2-5]. These studies are interesting in two respects. In one aspect, it would be interesting to explore the limits for extending the frequency range of acoustic oscillators. At ultrasonic frequencies, acoustic attenuation increases as frequency squared [6], hence the material $Q$ factor, i.e., the $Q$-factor due to material losses only, is inversely proportional to frequency. Considering that typical $Q$-factors at $\sim 1 \mathrm{GHz}$ at room temperature (RT) are on the order of 1000 [7], extrapolating this trend would mean bleak prospects for sub$\mathrm{THz}$ acoustic oscillators at RT. Fortunately, the trend does not continue into sub-THz frequencies due to the transition from the Akhiezer relaxation regime of phonon dissipation at ultrasonic frequencies to three-phonon scattering regime at $\mathrm{THz}$ frequencies [6, 8]. This transition is expected to yield a local maximum of material $Q$ in the sub-THz range [8]. Another interesting aspect is the connection to thermal transport studies. In Si at RT, most heat $(\sim 75 \%)$ is carried by phonons above $1 \mathrm{THz}$ in frequency [9]. However, sub-THz phonons are not entirely negligible: for example, the onset of the size effect in thermal conductivity of thin membranes is controlled by sub-THz phonon lifetime [10]. Also, we can use acoustic measurements in the sub$\mathrm{THz}$ range to test models used to analyze thermal transport in nanostructures, for example, models describing specular vs diffuse scattering at the boundaries [11].

Two most important characteristics of an acoustic oscillator are its frequency and damping time. The frequency of membrane thickness resonances in the sub-THz range is still well described by the elastic continuum model and given by $n c /(2 h)$, where $c$ is the speed of sound (longitudinal or transverse), $h$ is the membrane thickness and $n$ is a positive integer. In this study, our attention is focused on the damping time, or phonon lifetime, defined as a time in which the acoustic energy decays to the 1/e level. In a recent study [4], the lifetime of the fundamental (i.e., lowest frequency) longitudinal thickness resonance was measured in single crystal Si membranes in the thickness range 8-200 $\mathrm{nm}$ at RT. Scattering by surface roughness was identified as the main contributor to the observed decay rate of the acoustic oscillations, although this conclusion was made based on a comparison with model calculations of bulk 
phonon lifetimes rather than directly from the data. One complication in the analysis of the results of Ref. [4] arises from the fact that the frequency of the fundamental resonance depends on the membrane thickness, with the lifetime being affected by both thickness and frequency. It would be interesting to conduct a study including higher-order thickness resonances to look at the frequency dependence of the lifetime for a given membrane thickness, or, alternatively, at the thickness dependence of the lifetime at a given frequency. The former approach was pursued in Ref. [5] where the lifetime of thickness resonances of $\mathrm{Al} / \mathrm{Si}$ membranes in the frequency range 30-300 GHz was measured. The observed lifetimes were found to be strongly affected by the presence of the Al film.

In this work, we investigate the lifetime of high-order thickness resonances in a narrow frequency range around $\sim 270 \mathrm{GHz}$ on suspended single-crystal silicon membranes without a metal coating. The dominant role of scattering by surface roughness is established directly from the dependence of the measured lifetime on the membrane thickness. We quantify roughness using atomic force microscopy (AFM) and discuss the dependence of the lifetime on roughness for membranes of similar thickness. We also provide a lower bound for the intrinsic longitudinal phonon lifetime at $270 \mathrm{GHz}$ in silicon at RT.

\section{Samples}

Samples used in this study are listed in Table 1. All samples were single-crystal suspended Si membranes with the (100) surface orientation. The majority of the membranes were fabricated by etching silicon-on-insulator (SOI) wafers [12], while the two thicker samples were produced by polishing. The size of the free-standing area ranged from 0.5 to $5 \mathrm{~mm}$. Representative photographs of a few SOI-based samples are shown in Fig. 1. The membrane thickness was measured from the acoustic round-trip time as described in Sec. 3 below.

Surface roughness of all samples, with the exception of the two thinnest membranes, was measured by AFM, with the measurement results presented in Table 1. Figure 1 shows representative AFM images. With the exception of sample \#5, the root-mean-square (RMS) roughness was well under $1 \mathrm{~nm}$ and the lateral scale of topography features was under $\sim 50 \mathrm{~nm}$. Sample \#5, in contrast, had a large roughness with large lateral size features. Unfortunately, only one side of membranes was accessible to AFM measurements, as the supporting structure made the back side inaccessible to AFM due to geometric constraints. For polished samples, the same polishing procedure was applied to both sides, therefore the measurement are likely (albeit not 
guaranteed) to represent roughness on both sides of the sample. For membranes fabricated from SOI wafers, the etching process used on the front and back sides is not the same [12], therefore the back side roughness could be different.

\begin{tabular}{|l|l|l|l|l|l|l|}
\hline$\#$ & Thickness $(\mathrm{m})$ & Fabrication & Source & RMS roughness (nm) & $A_{2} / A_{1}$ & $Q$ \\
\hline 1 & 0.37 & SOI & in-house & & 0.55 & 120 \\
\hline 2 & 0.69 & SOI & in-house & & 0.40 & 150 \\
\hline 3 & 1.16 & SOI & commercial & 0.3 & 0.41 & 270 \\
\hline 4 & 1.49 & SOI & commercial & 0.2 & 0.43 & 350 \\
\hline 5 & 1.53 & SOI & in-house & 2 & 0.2 & 190 \\
\hline 6 & 1.69 & polished & commercial & 0.6 & 0.46 & 440 \\
\hline 7 & 15.3 & polished & commercial & 0.4 & 0.27 & 2400 \\
\hline
\end{tabular}

Table 1. List of samples and a summary of the measurements. $A_{2} / A_{1}$ is the amplitude ratio of the $2^{\text {nd }}$ and $1^{\text {st }}$ acoustic transients; $Q$ is the quality factor of the observed thickness resonances.

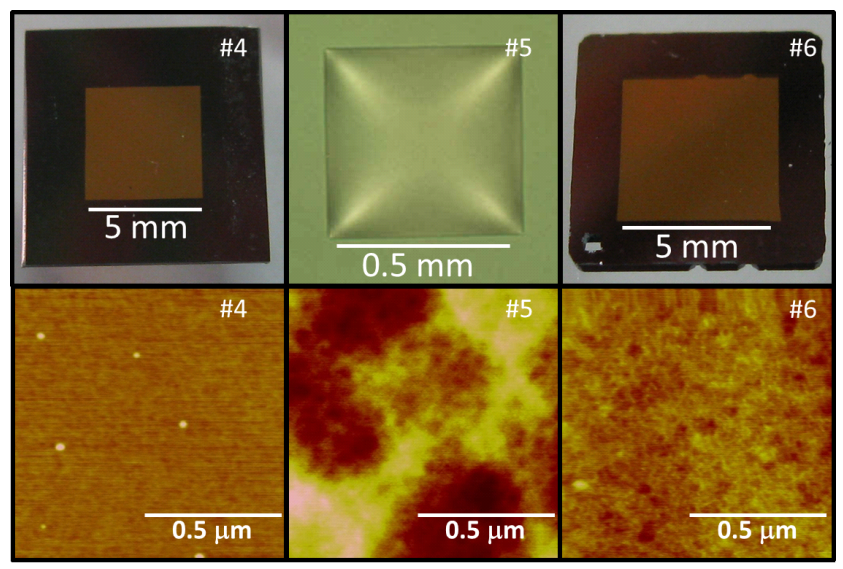

Fig. 1. Photographs and AFM images of samples \#4, \#5, and \#6. The RMS roughness is $0.2 \mathrm{~nm}$ for sample \#4, $2 \mathrm{~nm}$ for sample \#5, and $0.6 \mathrm{~nm}$ for sample \#6.

\section{Experiment}

The measurement configuration is shown schematically in Fig. 2(a). The frequencydoubled output of an amplified Ti:Sapphire system (wavelength $\lambda=387 \mathrm{~nm}$, pulse duration $300 \mathrm{fs}$, repetition rate $250 \mathrm{kHz}$ ) was split into excitation and variably delayed probe pulses. The excitation beam (pulse energy 30-80 nJ) was modulated by an acousto-optic modulator at $93 \mathrm{kHz}$ frequency to facilitate lock-in detection and focused to a spot of $50 \mathrm{~m}$ diameter (at 1/e intensity 
level) on one side of the sample, whereas the probe beam (pulse energy $\sim 2 \mathrm{~nJ}$ ) was focused to a $25 \mathrm{~m}$ diameter spot on the opposite side of the membrane.
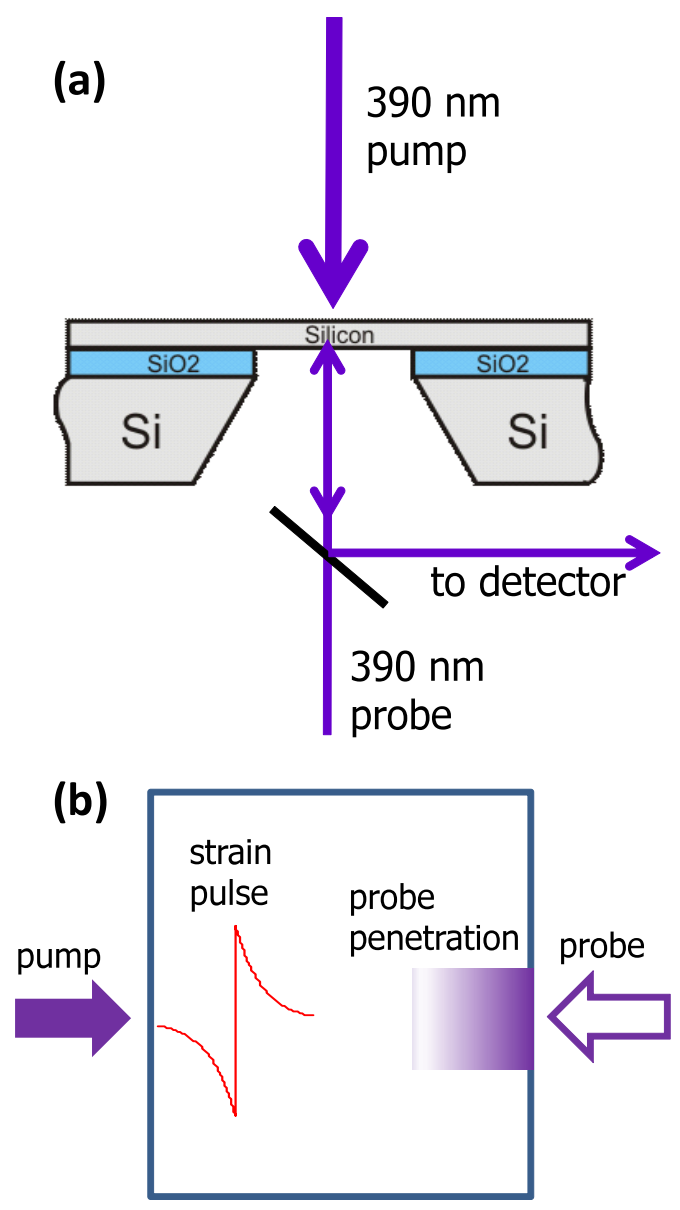

Fig. 2. (a) Schematic of the sample (with a SOI-based sample shown) and the measurement configuration. (b) Schematic of the excitation/detection process.

The excitation pulse generates a bi-polar strain pulse [13] with the pulse width corresponding to the penetration depth of excitation light $L=50 \mathrm{~nm}$ [14]. The strain pulse propagates towards the opposite surface of the sample, where it is reflected. When the strain pulse travels within the penetration depth of the probe light, the refractive index variation caused by the transient strain can be sensed by the probe pulse. The variation of the probe reflectivity as a function of the probe pulse delay for the $370 \mathrm{~nm}$ thick membrane is shown in Fig. 3(a). The slow signal is caused by diffusion of photoexcited carriers across the sample thickness and their subsequent recombination. On top of this slow signal one can see fast acoustic transients separated by equal intervals corresponding to the round-trip time inside the membrane $T=2 h / c_{\mathrm{L}}$, where $h$ is the membrane thickness and $c_{\mathrm{L}}=8433 \mathrm{~m} / \mathrm{s}$ [15] is the longitudinal acoustic velocity. 
The acoustic transients are dominated by fast oscillations at $\sim 270 \mathrm{GHz}$, which result from the interference of light reflected from the sharp step in the strain pulse and that reflected from the back surface of the sample [16,17]. Since the phase between the two interfering components shifts as the strain pulse propagates, the signal oscillates at the Brillouin frequency $f_{\mathrm{B}}=\lambda /\left(2 n c_{\mathrm{L}}\right)$, where $n$ is the refractive index. The refractive index of $\mathrm{Si}$ at $387 \mathrm{~nm}$ is 6.1 [14], resulting in a high Brillouin frequency [17]. The duration of the observed tone burst corresponds to the time during which the strain pulse remains within the penetration depth of the probe, i.e. $\sim 2 L / c_{L}=12$ ps. In fact the envelope of the acoustic signal captures the attenuation profile of the probe beam in the sample.
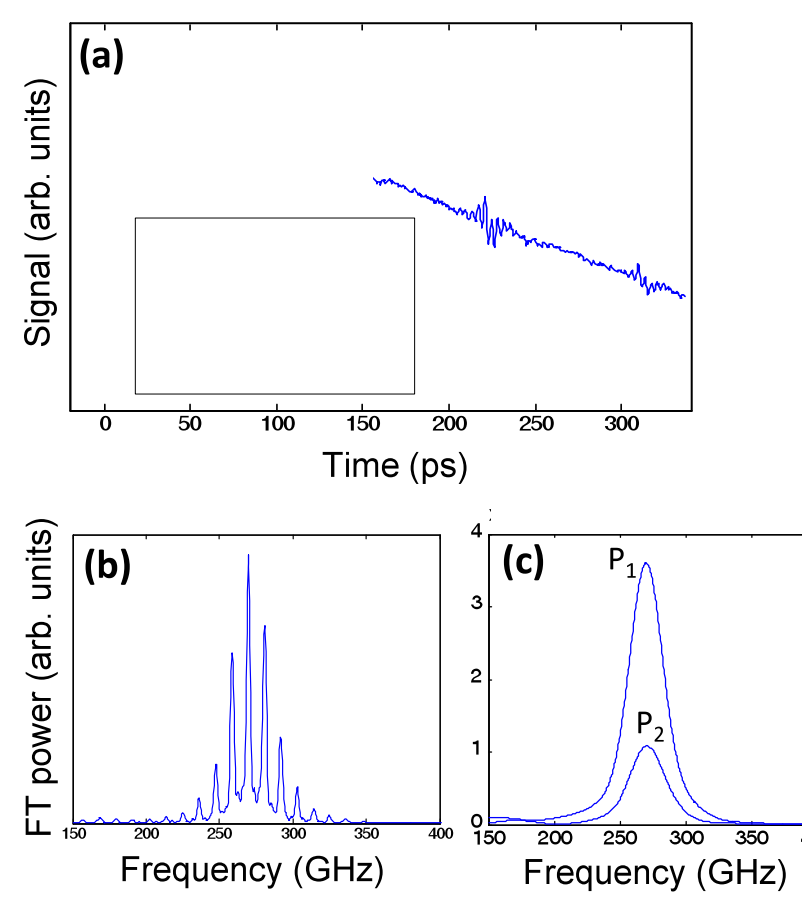

Fig. 3. (a) Transient reflectivity signal from the backside of the $380 \mathrm{~nm}$ membrane. The inset shows a close-up of the first acoustic transient with the slow background subtracted. (b) Fouriertransform of the acoustic signal. (c) Fourier-transforms of the first $\left(\mathrm{P}_{1}\right)$ and second $\left(\mathrm{P}_{2}\right)$ acoustic transients.

The Fourier-transform (FT) of the signal (taken after the slow background is subtracted) shown in Fig. 3(b) reveals a series of equally-spaced peaks corresponding to thickness resonances of the membrane $f_{n}=n c_{\mathrm{L}} / 2 h$, where $n$ is a positive integer indicating the mode number. The highest peak in the spectrum at $270 \mathrm{GHz}$ corresponds to $n=23$. The signal for thicker 
samples looks similar, with progressively longer delays between acoustic arrivals, and, correspondingly, smaller intervals between the thickness resonance modes. For the thickest 15 m sample the Brillouin frequency of $270 \mathrm{GHz}$ corresponded to very high order thickness resonances with $n \sim 900$. For such thick samples one may question the utility of describing the observed acoustic waves in terms of thickness resonances of a plate. Indeed, one can use an equivalent description in terms of bulk longitudinal waves bouncing back and forth between two surfaces. However, the FT of the signal does show discrete frequencies corresponding to thickness resonances of the sample as long as the time window used for the FT includes at least two acoustic arrivals. Exciting a single thickness resonance by a narrowband driving force would be in principle possible, even though this has not been done in our experiment. A related question is the validity of the plane wave representation, which implies disregarding the effect of the finite spot size. The latter becomes significant when the diffraction spread of the acoustic beam is comparable to the spot size, which happens at a propagation distance $\sim a^{2} / \lambda_{\mathrm{ac}}$, where $a$ is the excitation spot size and $\lambda_{\mathrm{ac}}$ is the acoustic wavelength equal to about $30 \mathrm{~nm}$. In our case this characteristic diffraction distance is $\sim 80 \mathrm{~mm}$, which greatly exceeds the propagation distance of a few round-trips even for the thickest sample. Consequently, the lateral spread of the acoustic energy in our experiment is entirely negligible.

Calculating Fourier-transform of each acoustic transient separately yields a broad peak centered at $\sim 270 \mathrm{GHz}$, as shown in Fig. 3(c). The magnitude of the FT peak diminishes after each round trip yielding nearly exponential decay, as shown in Fig. 4. The acoustic attenuation after one round trip was quantified based on the ratio of the amplitudes of the first and second acoustic transients, since those had the best signal-to-noise ratio. The amplitude attenuation was calculated from the ratio of the peak amplitudes $P_{\mathrm{i}}$ in the Fourier-transform power spectra as follows: $A_{2} / A_{1}=\left(P_{2} / P_{1}\right)^{1 / 2}$. The values of the amplitudes ratio $A_{2} / A_{1}$ are listed in Table 1 . The statistical error estimated by repeating the measurement at the same point on the sample ten times and calculating the standard error of the average value, was typically $\sim 2 \%$ and did not exceed $5 \%$ of the amplitudes ratio value. When more than one point on a sample was measured (see Fig. 5 below) the average value was entered in Table 1. 


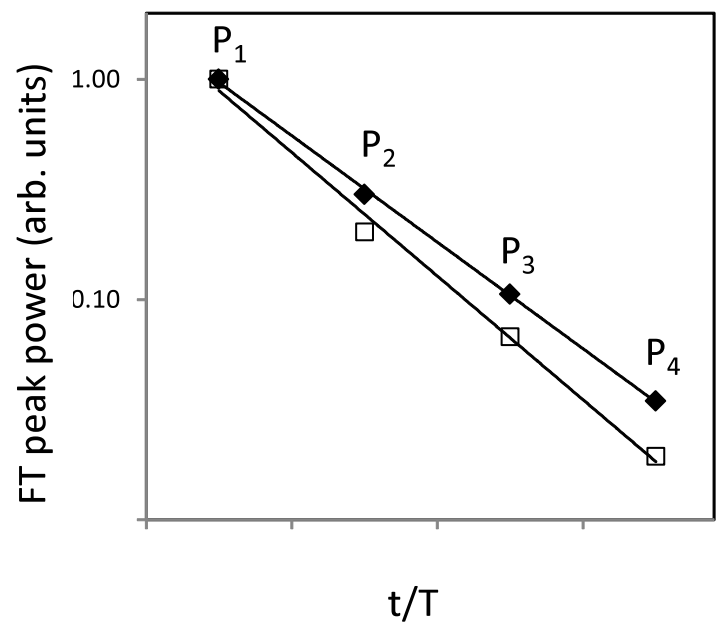

Fig. 4. FT peak magnitude of acoustic transients vs. their arrival time expressed in terms of the number of round-trips for sample \#1 (filled diamonds) and sample \#3 (open squares). The data have been normalized to yield unity for the first acoustic arrival and plotted on the logarithmic scale. Solid lines are exponential fits.

\section{Results and discussion}

From the amplitudes ratio $A_{2} / A_{1}$ and the round-trip time $T$, we calculate the effective phonon lifetime $\tau$. Assuming that the acoustic energy decays exponentially as $\exp (-t / \tau)$, the amplitude decay after one round trip will be given by $\exp (-T / 2 \tau)$, from which it follows that

$$
\tau=\frac{T}{2 \ln \left(\frac{A_{1}}{\left(A_{2}\right.}\right)} .
$$




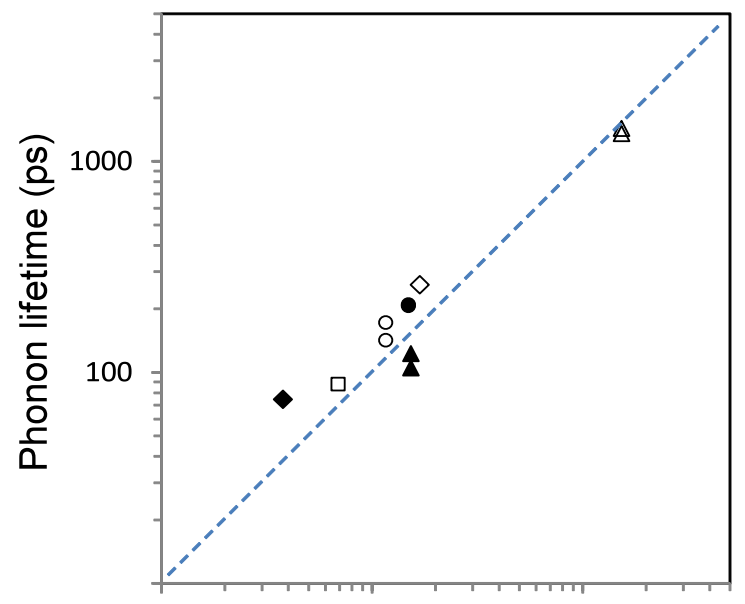

Sample thickness $(\mu \mathrm{m})$

Fig. 5. Phonon lifetime calculated according to Eq.(1) vs. sample thickness. Identical symbols correspond to measurements performed at different locations on the same sample, with two filled circles being too close to each other to be distinguishable. Statistical error for measurements at the same location is smaller than the symbol size. Dashed line with a slope of unity is a guide to the eye.

Figure 5 shows the dependence of the decay time on the sample thickness. One can see that the lifetime approximately scales with the thickness, although there are significant sampleto-sample variations for samples of approximately the same thickness. Both facts clearly indicate that the observed acoustic attenuation, with the possible exception of the thickest $15 \mathrm{~m}$ sample, is dominated by boundary scattering. If the attenuation was dominated by intrinsic bulk scattering, the lifetime would not depend on thickness. The lifetime of $1.4 \mathrm{~ns}$ measured on the 15 $\mathrm{m}$ sample gives us a lower bound for the intrinsic bulk phonon lifetime, which would correspond to a MFP of $13.5 \mathrm{~m}$. The lifetimes for other samples are 5 to 15 times smaller than this lower bound for the bulk lifetime; consequently, for those samples the bulk scattering can only play but a minor role. Significant sample-to-sample variations also point out to the dominant role of the boundary scattering. For example, out of three samples of similar thickness, $\# 4, \# 5$, and $\# 6$, sample \#5 yielded a much larger attenuation, which correlates with a significantly larger roughness of this sample.

Acoustic attenuation due to boundary scattering can be expressed in terms of surface specularity $p$, describing the fraction of the acoustic energy reflected from the surface in the specular direction, while the rest is lost to diffuse scattering. Assuming that the specularity of 
both surfaces of the sample is the same and bulk scattering is negligible, the acoustic energy reduction after a round trip, i.e., after two reflections, is given by $p^{2}$. Since the acoustic energy is proportional to the amplitude squared, we get $p^{2}=\left(A_{2} / A_{1}\right)^{2}$. Consequently, the specularity is equal to the amplitudes ratio,

$$
p=\frac{A_{2}}{A_{1}}
$$

As can be seen from Table 1, for the majority of the samples the measured specularity falls into a fairly narrow range $0.4-0.54$, the only exception being sample \#5 which has an exceptionally high roughness (the thickest $15 \mathrm{~m}$ sample is excluded from the consideration here because for that sample the contribution of the bulk scattering cannot be discounted). In the Kirchhoff approximation [18] which assumes that the correlation length of surface roughness is much greater than the acoustic wavelength and that the slopes of the surface are small, the specularity, in the case of normal incidence, is related to RMS roughness $\eta$ by an analytical formula

$$
p=\exp \left(-\frac{16 \pi^{2} \eta^{2}}{\lambda_{a c}^{2}}\right)
$$

In the solid state physics context this equation is often credited to Ziman [19], although it had been known previously [20]. Unfortunately, Ziman's book contains a mistake resulting in an extra $\pi$ in the equation, ${ }^{1}$ as has been noted in [21]. More importantly, Ziman does not provide a proper discussion of the domain of validity of Eq.(3) and the assumptions involved in its derivation; in fact, he implies that it is valid for small correlation lengths $l<<\lambda_{\mathrm{ac}}$ which is well known not to be the case $[18,20,22]$. Indeed, for deeply subwavelength scatterers one would expect the diffusely scattered power to scale as $\lambda_{a c}^{-4}$ whereas Eq.(3) yields $\lambda_{a c}^{-2}$ dependence. Despite the abundance of literature on wave scattering from rough surfaces (see [18, 22, 23], and references therein), a comprehensive quantitative theory of scattering of acoustic wave in a solid from a rough surface, even in the perturbation limit, appears to be still lacking. Thus we have to limit the comparison of our results with theory to a comparison with Eq. (3) while recognizing the limitations of the latter. ${ }^{2}$ Sample \#5 presents us with the only case of roughness with a large lateral feature size where Eq. (3) is expected to be valid. At $270 \mathrm{GHz}$, the longitudinal acoustic

\footnotetext{
${ }^{1}$ The extra $\pi$ first appears in Ziman's Eq. (6.9.14) and is then carried into Eq. (11.3.8) for the specularity parameter.

${ }^{2}$ An additional complication arising in the case of rough waveguides is the need to properly treat multiple scattering [22]. We believe that in our case treating scattering events as non-correlated [23] and applying the model developed for scattering of a bulk wave from a single surface is a reasonable approximation, although it may not work as well for fundamental thickness resonances [4].
} 
wavelength is $31 \mathrm{~nm}$; for the measured specularity of 0.2 Eq. (3) yields RMS roughness of $3.2 \mathrm{~nm}$. This is not too far from the experimentally measured RMS roughness value of $2 \mathrm{~nm}$, considering limited statistics provided by the AFM measurement on this sample. Therefore, we would suggest that in this case there is a reasonable agreement with Eq. (13).

The same cannot be said of samples \#3, \#4, and \#6, for which Eq. (3) predict RMS roughness between 2.2 and $2.3 \mathrm{~nm}$, whereas experimentally measured values range from 0.2 to $0.6 \mathrm{~nm}$. These samples have small-scale roughness with a correlation length comparable to or smaller than $\lambda_{a c}$, therefore Eq.(3) is not expected to apply. However, with the measured RMS roughness of just $1 / 100$ of $\lambda_{a c}$ or less (samples \#3 and \#4), a specularity of less than 0.5 does seem unlikely. We cannot exclude a possibility that roughness on the back side of these samples was larger than measured on the front side. Another possible explanation of a larger attenuation than expected based on surface roughness data would be inhomogeneous broadening due to thickness non-uniformity within the probe spot but on a larger scale than can be captured by AFM. However, inhomogeneous broadening decay, occurring as oscillators having different frequencies go out of phase [24], is unlikely to produce nearly exponential decay dynamics seen in Fig. 4.

It is instructive to express the phonon lifetime data in terms of the $Q$-factor of thickness resonances, $Q=\omega \tau$, where $\omega$ is the angular frequency. The $Q$-factor data are listed in the last column of Table 1. It is remarkable that the $Q$ factor for the thickest sample, which represents a lower bound for the intrinsic material $Q$-factor at $270 \mathrm{GHz}$ at RT, is as high as 2400 . In Fig. 6, the $Q$-factor for this sample is shown together with the bulk values obtained from the longitudinal acoustic lifetime data compiled in Ref. [8]. One can see that the measured $Q$-factor at $270 \mathrm{GHz}$ is almost as high as material $Q$ at $1 \mathrm{GHz}$. This indicates good prospects for the operation of acoustic devices at sub- $\mathrm{THz}$ frequencies at room temperature. However, the $Q$ factor of submicron membranes $\# 1$ and $\# 2$ is only on the order of 100 . Indeed, even in the absence of bulk losses, surface specularity limits the $Q$ factor to a value of

$$
Q_{p}=\frac{\pi n}{\ln (1 / p)},
$$

where $n$ is the thickness resonance order. The surface specularity would need to be significantly improved in order to achieve a high $Q$ in the fundamental or a low-order thickness resonance. 


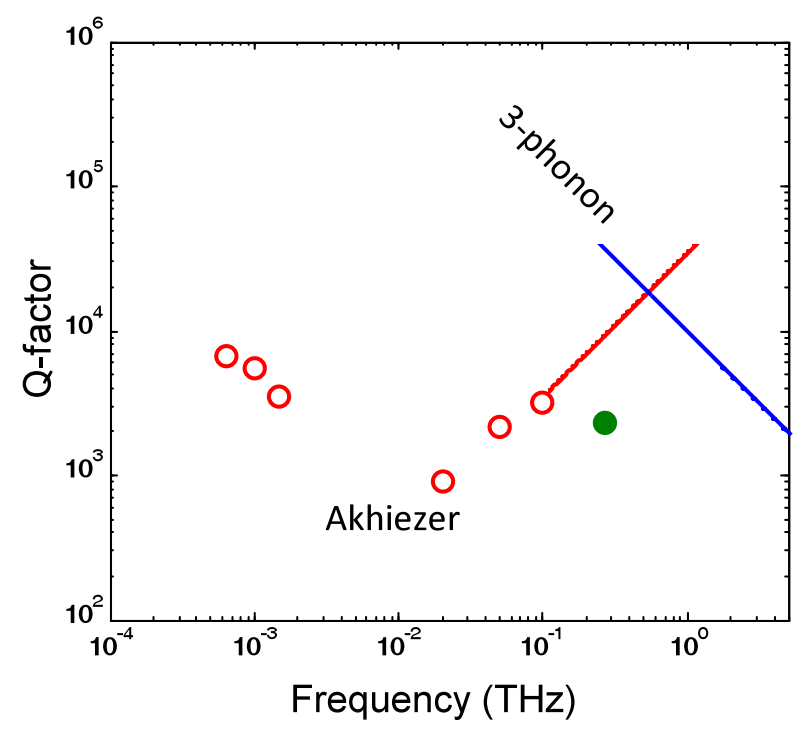

Fig. 6. $Q$-factor of thickness resonances of the $15 \mathrm{~m}$ sample from this work (solid symbol) and the intrinsic $Q$-factor of $\mathrm{Si}$ at RT from bulk lifetime data compiled in Ref. [8] (open symbols) fitted by the Akhiezer relaxation model. Also shown is a theoretical curve corresponding to the 3-phonon scattering model [9].

Let us now consider the implications of our surface specularity measurements for understanding thermal transport in thin Si membranes. The measured specularity at $270 \mathrm{GHz}$ does not exceed 0.55. Extrapolating to higher frequencies using Eq. (3) we find that at $1 \mathrm{THz}$ the specularity will not be expected to exceed $2 \times 10^{-4}$. Even considering the limited domain of validity of Eq. (3), it would be reasonable to suggest that the specularity will significantly drop at higher frequencies. Thus one would expect the diffuse boundary scattering model to work well at frequencies above $1 \mathrm{THz}$. Since above $1 \mathrm{THz}$ phonons carry about 75\% of heat in Si at RT [9], the diffuse boundary scattering model should be considered reasonable for analyzing thermal transport in Si membranes made from SOI wafers [12, 25].

\section{Conclusions}

Our measurements have indicated that the room temperature lifetime of high-order thickness resonances near $270 \mathrm{GHz}$ in $\mathrm{Si}$ membranes under $\sim 2 \mathrm{~m}$ in thickness is predominantly determined by surface roughness. A larger roughness has been shown to result in a reduced surface specularity, although the comprehensive understanding of the relationship between specularity and roughness requires further work on both theory and experiment. In this context, 
we would like to mention a connection to a recent study [26] where phonon transmission at $\sim 400$ $\mathrm{GHz}$ in Si nanosheets was found to be in the diffuse scattering regime, even though the measured surface roughness of $1 \mathrm{~nm}$ was expected to yield a significant specularity. Extrapolating our specularity measurements to higher frequencies we conclude that the "diffuse boundary scattering" model is a reasonable approximation in analyzing thermal transport in thin $\mathrm{Si}$ membranes. The measured $Q$-factors of thickness resonances reached 2400 in the $15 \mathrm{~m}$ sample confirming the existence of the region of high material $Q$ at $\mathrm{RT}$ at sub-THz frequencies. A low surface specularity will limit $Q$ factors achievable in nanoscale acoustic devices. Structures with atomically flat surfaces yielding a higher specularity at sub-GHz frequencies must be feasible: for example, Ref. [11] reported a specularity of 0.9 of a $\mathrm{GaN}_{-} \mathrm{SiO}_{2}$ interface at an acoustic frequency of $425 \mathrm{GHz}$. However, a submicrometer-thick resonator may need an even higher specularity to take the full advantage of the high material $Q$.

\section{Acknowledgements}

This work was supported as part of the S3TEC Energy Frontier Research Center funded by the U.S. Department of Energy, Office of Basic Energy Sciences under Award No. DESC0001299/DE-FG02-09ER46577. The authors would like to thank Clivia M. Sotomayor and Gang Chen for stimulating discussions. C. M. Sotomayor and Timothy Kehoe are also thanked for their help in fabricating the in-house samples, which were fabricated at the Catalan Institute

for Nanoscience and Nanotechnology using facilities of the ICTS "Integrated Nano and Microfabrication Clean Room”, (CSIC-CNM).

\section{References}

[1] K. M. Lakin, IEEE Trans. Ultrason. Ferroelect. Freq. Control 52, 707 (2005).

[2] F. Hudert, A. Bruchhausen, D. Issenmann, O. Schecker, R. Waitz, A. Erbe, E. Scheer, T. Dekorsy, A. Mlayah, and J.-R. Huntzinger, Phys. Rev. B 79, 201307 (2009).

[3] M. Harb, W. Peng, G. Sciaini, C. T. Hebeisen, R. Ernstorfer, M. A. Eriksson, M. G. Lagally, S. G. Kruglik, and R. J. D. Miller, Phys. Rev. B 79, 094301 (2009).

[4] J. Cuffe, O. Ristow, E. Chávez, A. Shchepetov, P.-O. Chapuis, F. Alzina, M. Hettich, M. Prunnila, J. Ahopelto, T. Dekorsy, C. M. Sotomayor Torres, Phys. Rev. Lett. 110, 095503 (2013). 
[5] M. Grossmann, M. Klingele, P. Scheel, O. Ristow, M. Hettich, C. He, R. Waitz, M. Schubert, A. Bruchhausen, V. Gusev, E. Scheer, and T. Dekorsy, Phys. Rev. B 88, 205202 (2013).

[6] H. J. Maris, in Physical Acoustics, edited by W. P. Mason and R. N. Thurston (Academic, New York, 1971), Vol. 8, p. 279.

[7] H. P. Loebl, M. Klee, C. Metzmacher, W. Brand, R. Milsom, P. Lok, Materials Chemistry and Physics 79, 143 (2003).

[8] B. C. Daly, K. Kang, Y. Wang, and D. G. Cahill, Phys. Rev. B 80, 174112 (2009).

[9] A. Ward, D.A. Broido, Phys. Rev. B 81, 085205 (2010).

[10] A.A. Maznev, J. Appl. Phys., 113, 113511 (2013).

[11] Y.-C. Wen, C.-L. Hsieh, K.-H. Lin, H.-P. Chen, S.-C. Chin, C.-L. Hsiao, Y.-T. Lin, C.-S. Chang, Y.-C. Chang, L.-W. Tu, C.-K. Sun, Phys. Rev. Lett. 103, 264301 (2009).

[12] A. Johnson, A. A. Maznev, J. Cuffe, J. K. Eliason, A. J. Minnich, T. Kehoe, C. M. Sotomayor Torres, G. Chen, and K. A. Nelson, Phys. Rev. Lett. 110, 025901 (2013).

[13] V. E. Gusev and A. A. Karabutov, Laser Optoacoustics (American Institute of Physics, 1993).

[14] Handbook of Optical Constants of Solids, Vol. 3, edited by E.D. Palik (Academic Press, 1998).

[15] H. J. McSkimin and P. Andreatch Jr., J. Appl. Phys. 35, 2161 (1964);

[16] H. N. Lin, R. J. Stoner, H. J. Maris, and J. Tauc, J. Appl. Phys. 69, 3816 (1991).

[17] A. Devos, M. Foret, S. Ayrinhac, P. Emery, and B. Rufflé, Phys. Rev. B 77, 100201 (2008).

[18] J. A. Ogilvy, Rep. Prog. Phys. 50, 1553 (1987).

[19] J. M. Ziman, Electrons and Phonons (Clarendon Press, Oxford, 1960).

[20] S. O. Rice, Comm. Pure Appl. Math. 4, 351 (1951).

[21] Z. M. Zhang, Nano/Microscale Heat Transfer (McGraw-Hill, 2007).

[22] F. G. Bass and I. M. Fuks, Wave Scattering from Rough Surfaces (Pergamon Press, Oxford, 1979).

[23] A. G. Voronovich, Wave Scattering from Rough Surfaces (Springer, Berlin, 1999).

[24] A. M. Weiner, Ultrafast Optics (Wiley, 2009).

[25] A. M. Marconnet, M. Asheghi, K. E. Goodson, ASME J. Heat Transfer 135, 061601 (2013).

[26] J. B. Hertzberg, M. Aksit, O. O. Otelaja, D. A. Stewart, and R. D. Robinson, Nanolett. 14, 403 (2014). 